\title{
ELENCHOS
}

Rivista di studi sul pensiero antico fondata da

Gabriele Giannantoni

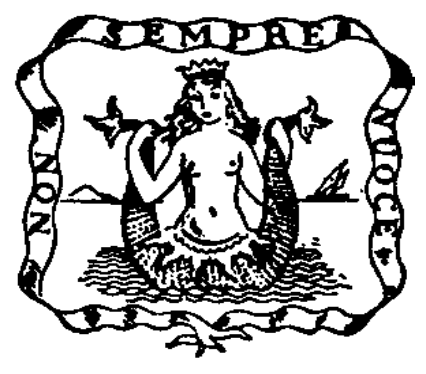

BIBLIOPOLIS 
«Elenchos». Rivista di studi sul pensiero antico

fondata da Gabriele Giannantoni

a cura dell'Istituto per il Lessico Intellettuale Europeo e Storia delle Idee.

Sezione Pensiero Antico. C.N.R.

Direttore: Anna Maria Ioppolo

\section{COMITATO DIRETTIVO:}

Francesco Adorno, Enrico Berti, Aldo Brancacci, Fernanda Decleva Caizzi, Barbara Faes de Mottoni, Anna Maria Ioppolo, Giovanni Reale, Carlo Augusto Viano

RESPONSABILE DI REDAZIONE:

Maria Cristina Dalfino

COMITATO DI REDAZIONE:

Michele Alessandrelli, Riccardo Chiaradonna, Alberto Manchi, Luca Simeoni

EDITING:

Maria Cristina Dalfino

La Direzione di «Elenchos» ha sede presso l'Istituto per il Lessico Intellettuale Europeo e Storia delle Idee. Sezione Pensiero Antico, Villa Mirafiori, via C. Fea 2, 00161 Roma. http://www.iliesi.cnr.it

elenchos@rmcisadu.let.uniroma1.it

I manoscritti vanno indirizzati ad Anna Maria Ioppolo, presso l'Istituto.

Ai dattiloscritti devono essere allegate copie su dischetto (Word per Mac o Pc; font Supergreek per il greco; formato RTF per l'invio in allegato)

Periodici e pubblicazioni vanno indirizzati alla Direzione di «Elenchos».

L'amministrazione di «Elenchos» ha sede presso la casa editrice «Bibliopolis, edizioni di filosofia e scienze di Francesco del Franco», 80122 Napoli, via Arangio Ruiz 83. www.bibliopolis.it

info@bibliopolis.it

«Elenchos» ha periodicità semestrale ed è pubblicata nei mesi di maggio e novembre.

Abbonamenti: per l'Italia: $€ 21,00$

per l'estero: $€ 26,00$

Un fascicolo: per l'Italia: $€ 13,00$

per l'estero: $€ 16,00$

I versamenti vanno effettuati sul c.c.p. n. 74019035 intestato a: «BIBLIOPOLIS di Francesco del Franco», Napoli, via Arangio Ruiz 83.

Autorizzazione del Tribunale di Napoli n. 5805/80

Direttore responsabile: Anna Maria Ioppolo 